\title{
Gambling Disorder and suicidality within the UK: An analysis investigating mental health and gambling severity as risk factors to suicidality
}

\section{Abstract}

Greater severities of gambling behaviour and poorer mental health have been identified in the literature as risk factors for suicidality within gambling disorder (GD) populations. This is the first known study within the UK to use empirical data, taken from a UK treatment clinic for GD ([removed for peer review]) to explore the associations between current suicidality (thoughts and plans) and measures assessing gambling severity and mental health. Self-report data from 122 participants were collected during the intake process at the clinic through a variety of methods including the use of questionnaires and a standardised 90-minute interview with a psychologist. Reported suicidality was high (current suicidal thoughts [28.7\%] and plans [6.6\%]), yet only one measure of gambling severity (estimated total losses) was found to be inversely associated with suicidality. Indices of poorer mental health were found to have a greater association with suicidality than measures of gambling severity, and a logistic regression analysis identified depressive symptomology and a history of psychiatric disorder in the participants' family as significant factors associated with suicidality. Overall, within GD populations, factors associated with poorer mental health rather than gambling severity are seemingly a greater risk factor in patients with suicidality.

Keywords: Gambling Disorder, Suicidality, Suicidal Ideation, Gambling Severity, Mental Health. 


\section{Introduction \& Background}

Prevalence statistics for the general population identify rates of suicidal thoughts as being between $2.8-18.5 \%$, suicidal plans, $0.7 \%-3 \%$ and suicidal behaviour, 0.4\%-5\% (Kessler et al., 1999; Kessler et al., 2005; Weissman et al., 1999). Within the UK, the prevalence of suicidal thoughts has been found to be $2.3 \%$ (Gunnell et al. 2004) and the suicide rate is approximately 10.8 per 100,000 (Scowcroft, 2016). Suicide currently accounts for $1.5 \%$ of all deaths and is the tenth leading cause of all deaths worldwide (Hawton \& Heeringen, 2009). This phenomenal statistic therefore emphasises the need for a developed awareness regarding suicidality and the associated risk factors within differing populations. The most recent UK prevalence survey identified $0.9 \%$ of the population to be affected by Gambling Disorder (GD) (Wardle et al., 2010). The pervasiveness of GD necessitates exploration into the possible associated risks, including suicidality.

Research has identified GD populations to have higher rates of suicidality compared to the general population. Rates of suicidal ideation have been identified as being between $13 \%$ and $81.4 \%$ and rates of suicide attempt, 2.3\%-30.2\% (Battersby et al., 2006; Frank et al., 1991; McCallum \& Blaszczynski, 2003; McCormick et al., 1984; Petry \& Kulik, 2002). Most recently, within a Singaporean treatment-seeking GD population, 37\% reported current suicidal thoughts, $16 \%$ current suicidal plans and $11 \%$ reported a lifetime history of suicide attempts (Manning et al., 2015).

While there is variability within these rates, potentially due to the seemingly increased risk of suicidality within GD populations, it has been argued that the severity of an individual's gambling behaviour may be associated with a heightened risk of suicidality. Suicidality within a GD population has been found to be associated with a greater number of behavioural manifestations of GD (Petry \& Kulik, 2002; Hodgins, Mansley \& Thygesen, 2006). The research thus emphasizes the potential role of gambling behaviour within suicidality and indicates gambling severity as a feature relating to an increased risk of suicidality within GD populations.

The enduring link found between mental health and suicidality within the general population provokes enquiry as to whether this association would also be present within GD populations. Suicidality risk factors within the general population include: a familial history of psychiatric disorder (Brent et al., 1994; Qin et al., 2002), previous suicide attempts and a previous psychiatric diagnosis (Chan, Shamsul \& Maniam, 2014; Harris \& Barraclough, 1997; Nock et al., 2008; Oquendo, Currier \& Mann, 2006; Ruengorn et al., 2011; Windfuhr \& Kapur, 2011).

High levels of psychiatric disorder have been found to be present within GD populations. Recent studied have identified levels of current and lifetime comorbid psychiatric disorders to be high $(74.8 \%$ and $75.5 \%$ respectively; Dowling et al. 2015), with GD populations exhibiting particularly elevated 
levels of previous treatment for mood disorder and anxiety disorder (lifetime: $50 \%$ and $42 \%$, respectively; Toneatto \& Pillai, 2016). Furthermore, research has also identified increased levels of depression (Becona, Lorenzo \& Fuentes, 2006; Kim et al., 2006; Specker et al., 1996), high levels of previous treatment for a psychiatric disorder, 55\% (Battersby et al., 2006), high levels of a previous psychiatric diagnosis, $60 \%$, as well as a greater likelihood of reporting a personality disorder in GD populations when compared to the general population (Séguin et al., 2010).Thus, it has been hypothesised that that preceding poor mental health contributes to the onset of GD (Blaszczynski, \& Nower, 2002). This is seemingly supported by the prevalence of GD within individuals with a psychiatric diagnosis being between $6 \%$ and $12 \%$ (George \& Murali, 2005). Within a large-scale study $(\mathrm{n}=7214)$, Newman and Thompson (2003) also identified GD as being associated with suicidality and suggested that this relationship was owing to poor mental health, thus further emphasising the role of mental health within GD.

This proposition, the association within general population studies between mental health and suicidality, the prevalence of psychiatric disorder identified within GD populations and the role of gambling behaviour identified within suicidality, provoke exploration into the relationship between gambling behaviour, mental health and suicidality in GD populations. Both poor mental health and gambling disorder have been identified within the literature as factor associated with high rates of suicidality in the GD population. Hodgins et al. (2006) identified suicidality to be associated with higher levels of gambling severity within a Canadian GD population, overall, the study identified indices of poorer mental health to be more greatly associated with suicidality; in particular the association between depression and suicidality was highlighted. Similarly, Manning et al. (2015) identified debt as being the second most pertinent variable correlated with suicidality in the sample, this ostensibly a measure of gambling severity. However, psychiatric disorder was identified as being most strongly associated with suicidality.

This study aims to identify the relative factors associated with suicidality within a UK GD population. As far as the authors are aware, this study is the first to investigate the associations between gambling severity, mental health and suicidality in a UK treatment-seeking GD population. To address this knowledge gap, measures of current suicidality (thoughts and plans) were investigated to provide a comprehensive account of suicidality within a UK GD treatment-seeking population.

\section{Method}

\subsection{Participants}

The data used in this study was collected from a pre-existing anonymised expanding database of routine clinically collected data from patients who were seeking treatment for GD at the [removed for peer review]. The [removed for peer review], based in London, is the only [removed for peer review] for GD 
patients in the UK. As a national clinic, the [removed for peer review] receives referrals from all over the UK. Data for 122 participants (110 males) was collected between September 2014 and January 2015. Participants were inclusive of those individuals who registered with the clinic and completed an initial assessment during this time period. The mean age of the sample was 35.5 years $(S D=11.40)$. All participants met the DSM-5 criteria for GD (APA, 2013). The Problem Gambling Severity Index (PGSI) (Ferris \& Wynne, 2001), scored on a scale of $0-27$, was used within the study as one measure of gambling severity. Participants had a mean PGSI score of 19.84 (SD=4.63).

\subsection{Materials}

Self-report measures in the form of a referral form, a health questionnaire, and a comprehensive clinical interview were used in data collection. The referral form was used to request an appointment for assessment with the [removed for peer review] and provided demographic information for each participant as well as gambling severity indicators.

The health questionnaire included, the Patient Health Questionnaire (PHQ-9) used to assess the severity of depression (Scored on a scale of 0-27) (Kroenke at al., 2001), the PGSI (Ferris \& Wynne, 2001), the GAD-7 - an assessment of Generalised Anxiety Disorder (scored on a scale of 0-21) (Spitzer et al., 2006) - as well as a subjective rating of 'mental health', 'physical health' and 'quality of life' on a scale of $0-20$, which formed part of a treatment outcome profile (TOP).

Data was also collected from a standardised 90-minute semi-structured clinical interview undertaken by a psychologist. In this interview, data was recorded on a standardised form. This interview allowed for the collection of a large amount of data including information regarding participants' gambling behaviour, whether they had experienced an early big win, their psychiatric history as well as information relating to suicidality, medical history, forensic history, family psychiatric history, family support network, employment history and personal history. The DSM-5 criteria were also used to establish a participant's diagnosis of GD (APA, 2013). Table 1 provides an overview of the categorisation and timeframes used within the assessment to establish levels of suicidality. No current suicidal behaviours were reported and therefore the relationships between current suicidal behaviour and the established measures for gambling behaviour and mental health were not explored within this study and suicidality refers to a composite measure formulated of both reported suicidal thoughts and plans.

\subsection{Design}

Cross-sectional data were used to assess the associations between current suicidality (referenced as thoughts and plans - there was no suicidal behaviour within the sample), measures of gambling behaviour (used to assess gambling severity) and mental health status. A range of criteria is used to assess both gambling severity and mental health, these measures are outlined in Table 2 and 3.

$<$ Insert Table 1> 


\subsection{Procedure}

[Removed for peer review] ethics committee approved this study. The use of routine clinically collected data was also validated under the [removed for peer review] Research Authority ([removed for peer review]) associated with the [removed for peer review], UK. All data were anonymised to the researchers within this study.

Participants completed a referral form, accessible to the general population via the treatment clinic's website or via referral agents. Following this, the participants attended an assessment at the treatment clinic in which they completed a health questionnaire and undertook an interview with a psychologist. All data was collected using standardised assessment forms.

\subsection{Statistical Analysis}

Differences between mental health outcomes, gambling severity and age in participants who did or who did not report any suicidality (thoughts and plans) were analysed using independent samples t-tests. For data analysis purposes, all suicidal participants [thoughts and plans] were grouped together and independent sample t-tests were used to compare suicidal vs. nonsuicidal GD participants on indices of gambling severity and mental health outcomes. Logistic regression analysis was used to identify the most prominent factors associated with suicidality and to identify if poorer mental health or gambling severity increases the risk of suicidality within a GD population. A correlation matrix was used to aid with the selection of variables within the logistic regression to avoid multicollinearity. Those variable significantly associated $(p<.05)$ were scrutinized for inclusion in the logistic regression model. A threshold for collinearity was set at $>0.7$ (Dormann et al. 1994) however, both the GAD-7 and the PHQ-9 were retained within the analysis as validated measures of distinct constructs (Beuke et al., 2003). See Table 2. All data were analysed using SPSS Version 22.0 (IBM Corp, 2013).

$<$ Insert Table 2>

\section{Results}

\subsection{Descriptive statistics of the study population}

Males represented $90.2 \%$ of the study population. The self-reported average age of onset of GD was 23.6 years $(S D=10.2) .31 .1 \%$ of the sample reported a familial history of GD. 27.9\% reported loss of a relationship due to their gambling behaviour and 18\% reported a loss of employment due to their gambling behaviour. $36.9 \%$ reported a previous diagnosis of a co-morbid psychiatric disorder. The mean PHQ-9 score of the sample was 12.8 ( $S D=$ 7.6) and the mean GAD-7 score, $10.7(S D=6.1)$. With reference to suicidality, 35 participants (28.7\%) reported current suicidal thoughts and within this sample 8 participants also (6.6\%) reported current suicidal plans.

$<$ Insert Table 3> 


\subsection{Relationships between suicidality and gambling severity}

The relationship between whether a participant reported suicidal thoughts or plans and the severity is reported in Table 3 . There was a significant difference between the group reporting suicidality and those not reporting suicidality regarding estimation of total losses (estimated total monetary losses-lifetime [£]). Those with suicidality reported a smaller estimated loss. As can be seen in Table 3, there were no other significant relationships between suicidality and indices in gambling behaviour.

\subsection{Relationships between suicidality and mental health symptom severity measures}

Table 3 also shows the associations explored between mental health symptom severity measures and reported suicidality. Participants who reported suicidality reported poor mental health, poorer physical health, worse 'quality of life' status, higher depression, and higher levels of anxiety.

\subsection{Regression Analysis}

The predictor variables: no. DSM-5 criteria, age of onset of GD, experience of an early big win, current debt (as a median split analysis), estimated total losses (as a median split analysis), PHQ-9 score at assessment, GAD-7 score at assessment, presence of a psychiatric history within family members, the presence of a psychiatric comorbidity and no. days gambled prior to assessment [as a median split analysis], were selected and entered into a logistic regression analyses to establish the most significant factors associated with suicidality. Measures of gambling severity (PGSI and No. DSM-5 criteria) were not found to be significantly correlated within this sample (see table 2).

PHQ-9 score at initial assessment and the presence of a family psychiatric history were found to be significantly associated with suicidal thoughts, (OR: 1.19, Cl: 1.03, 1.38, $p=.02$; OR: 3.02, Cl: 1.05, 8.69, $p=.04$, respectively). See Table 4.

$<$ Insert Table 4>

\section{Discussion}

This is the first UK study to explore the associations between gambling severity, mental health and suicidality in a treatment-seeking GD population. Supporting the hypothesis of this study, indices of poorer mental health rather than gambling severity were found to be more greatly associated with suicidality. Furthermore, an independent samples t-test revealed that smaller estimated total losses were found to be associated with suicidality. Measures associated with mental health were found to be more strongly correlated with suicidality. This was further supported by a logistic regression analysis identifying higher levels of depression and a family psychiatric history as being significantly associated with suicidality. 
This study supports previous prevalence statistics for suicidality within GD populations within other countered (Frank et al., 1991; Mccallum \& Blaszczynski, 2003; McCormick et al., 1984; Petry \& Kiluk, 2002) and substantiates gambling disorder as a risk factor associated with suicidality. An association between suicidality and estimated total losses was found. However, surprisingly, the direction of this association is contradictory to previous research as smaller estimates of losses, rather than larger losses, were found to be more greatly associated with current suicidality. Blaszczynski and Farrell (1998) identified acute financial loss as a risk factor for suicide within GD populations. Greater financial loss has also been identified as a risk factor for suicide in general population studies (Cheng et al., 2000; Pridmore \& Reddy, 2012). There are several explanations that can be offered for this finding that is contradictory to previous literature. One possible reason is that if underlying pathology in mood is fundamental to the manifestation of suicidality, then there is no reason to expect increased suicidality to be associated with markers that suggest greater problems with pathological gambling. Also of all the measures used, estimated total losses is the most subjective measure of gambling severity and may tap into something other than severity, such as a desire to come across as more severe or less severe, perhaps in this context due to acceptability biases, social desirability or in hope of altering treatment waiting times. It should also be noted that within a population affected by psychiatric/psychological issues (who may not have an income or be unable to work compared to their peers in better mental health [Kessler et al., 2001]), any losses may be highly detrimental. Furthermore, small monetary losses in an individual within poor resilience due to mental health issues may also be perceived as highly damaging. Thus, while this finding is contradictory to previous studies, the potential context of this finding must remain a consideration. Finally, as the inverse relationship between total losses and suicidality only emerged in the univariate analysis and not the multivariate analysis it is possible that this association is an incidental finding or that it is influenced by one or more other factors that were only included in the multivariate model such as age of onset of GD or potentially reported current debt. At present further exploration is required to understand this finding.

In support of previous research, poorer mental health rather than gambling severity was found to be a prominent feature of suicidality within GD (Mccallum \& Blaszczynski, 2003; Manning et al., 2015; Hodgins et al., 2006; Thon et al., 2014). The limited number of gambling severity outcomes found to be associated with suicidality does however differ to previous studies identifying gambling severity as a greater feature of suicidality (Frank et al., 1991; Petry \& Kiluk, 2002; Hodgins et al., 2006; Ledgerwood et al., 2004), which further supports the suggestion that poorer mental health is more greatly associated with suicidality than gambling severity within GD populations.

The prominence of mental health, particularly higher levels of depression, with regard to suicidal thoughts, supports MacCallum and Blaszczynski (2003) 
who found depression to be associated with both current and past suicidality within a GD population. This finding also seemingly supports literature highlighting the role of depression within GD (Becona et al., 2006; Kim et al., 2006; Specker et al., 2006). The association identified between a familial psychiatric diagnosis and suicidality highlights the need for risk factors within the general population (Brent et al., 1994; Qin et al., 2002) to still be considered within GD populations.

A lack of association between the measures of gambling severity (PGSI and no.DSM-5 criteria) also merits comment. This finding seemingly supports suggestions by Wardle et al. (2010) that while both are used as a measure of gambling severity they remain distinctly different. British prevalence rates were found to differ between the two measures (PGSI: 0.6\%; DSM-5: 0.9\%; Wardle et al., 2010) and Griffiths (2014) highlights the notion that these measures maybe capturing differing gambling related issues or even differing individuals. Furthermore, the PGSI has only been validated within Canadian populations and thus results may differ within a British population.

\subsection{Practical Impact}

Principally, this study highlights the risk of suicidality within GD. While phenomena such as suicide cannot be reliably predicted by clinicians (Rudd \& Joiner, 1998), this study emphasises the need for clinicians to be cognisant of the risk of suicidality and implement appropriate assessment and prevention approaches within the treatment of GD, e.g. the use of brief intervention strategies (Fleischmann et al., 2008; Stanley \& Brown, 2012). The findings of this study also suggest that mental health rather than gambling severity should be addressed initially within treatment to ensure the enhancement of treatment outcomes regarding suicidality.

While the presence of risk factors should provoke clinicians to acknowledge the risk of suicide, the complex aetiology of GD and general population risk factors for suicide also highlight the need for rigorous screening and continual monitoring of suicidality throughout treatment. It is necessary for suicide to be acknowledged as a risk across all GD populations to aid in the reduction of suicidal behaviour.

\subsection{Limitations}

This study only included an outpatient treatment-seeking population and thus findings cannot be generalised across all GD populations, as sample characteristics may differ within alternative populations, i.e. those who do not seek treatment.

No current suicidal behaviours were reported within the sample thus the relationship between current suicidal behaviour, mental health and gambling severity could not be investigated. To provide a more comprehensive account of the association between mental health, gambling severity and current suicidality, further research that is inclusive of this data is required. 


\subsection{Recommendations for future studies}

The role of depression and psychiatric disorder highlighted within this study, as well as research identifying depression as a potential contributing factor to the onset of GD (Blaszczynski \& Nower, 2002) elicits further investigation into the relationship between mental health, GD and suicidality as well as the temporal ordering between GD and depression. Additional investigations within this area are required to augment awareness of the risk factors associated with GD and enhance treatment programmes and clinical practice.

\subsection{Conclusion}

This study was the first in the UK to explore the associations between mental health, gambling behaviour and suicidality in a treatment-seeking GD population. The rates of suicidal ideation identified further substantiate suicide as a risk factor within GD and support previous literature identifying mental health rather than gambling severity as a greater predictor suicidality. Poorer mental health was found to be more greatly associated with suicidal thoughts and plans. Higher levels of depression were found to be a significant predictor of suicidality and a history of psychiatric disorder in the family was found to be a significant predictor of suicidality. This study identifies the need for the implementation of rigorous assessment and prevention strategies for suicidality within the treatment of GD and necessitates research to identify the causal relationship between poor mental health, GD and suicidality to enhance awareness and clinical treatment. However, provisionally, there is a need for clinicians to be aware of suicide risk within GD populations and its predictors, particularly depression and psychiatric disorder. 


\section{References}

American Psychiatric Association [APA] (2013). Diagnostic and statistical manual of mental disorders (5th edn). Arlington, VA: American Psychiatric Publishing.

Battersby, M., Tolchard, B., Scurrah, M. \& Thomas, L. (2006). Suicide ideation and behaviour in people with pathological gambling attending a treatment service. International Journal of Mental Health and Addiction, 4, 233-246.

Becona, E., Lorenzo, M. D. C. \& Fuentes, M.J. (1996). Pathological gambling and depression. Psychological Reports, 78, 635-640.

Beuke, C. J., Fischer, R., \& McDowall, J. (2003). Anxiety and depression: Why and how to measure their separate effects. Clinical psychology review, 23(6), 831-848.

Blaszczynski, A. \& Farrell, E. (1998). A case series of 44 completed gambling-related suicides. Journal of Gambling Studies, 14(2), 93-109.

Blaszczynski, A. \& Nower, L. (2002). A pathways model of problem and pathological gambling. Addiction, 97, 487-499.

Brent, D. A., Perper, J. A., Moritz, G., Liotus, L., Schweers, J., Balach, L., \& Roth, C. (1994). Familial risk factors for adolescent suicide: a casecontrol study. Acta Psychiatrica Scandinavica, 89(1), 52-58.

Chan, L. F., Shamsul, A. S. \& Maniam, T. (2014). Are predictors of future suicide attempts and the transition from suicidal ideation to suicide attempts shared or distinct: A 12-month prospective study among patients with depressive disorders. Psychiatry Research, 220(3), 867-873.

Cheng, A. T., Chen, T. H., Chen, C. C., \& Jenkins, R. (2000).

Psychosocial and psychiatric risk factors for suicide: Case-control psychological autopsy study. The British Journal of Psychiatry, 177(4), 360-365.

Dormann, C. F., Elith, J., Bacher, S., Buchmann, C., Carl, G., Carré, G., ... \& Münkemüller, T. (2013). Collinearity: a review of methods to deal with it and a simulation study evaluating their performance. Ecography, 36(1), 27-46.

Dowling, N.A., Cowlishaw, S., Jackson, A.C., Merkouris, S.S., Francis, K.L. \& Christensen, D.R. (2015). Prevalence of psychiatric co-morbidity in treatment-seeking problem gamblers: A systematic review and metaanalysis. Australian and New Zealand Journal of Psychiatry, 49(6), 519539. 
Ferris, J. \& Wynne, H. (2001). The Canadian problem gambling index: Final Report. Ottawa, ON: Canadian Centre on Substance Abuse.

Fleischmann, A., Bertolote, J. M., Wasserman, D., De Leo, D., Bolhari, J., Botega, N. J., et al. (2008). Effectiveness of brief intervention and contact for suicide attempters: a randomized controlled trial in five countries. Bulletin of the World Health Organization, 86(9), 703-709.

Frank, M. L., Lester, D. \& Wexler, A. (1991). Suicidal behavior among members of Gamblers Anonymous. Journal of Gambling Studies, 7(3), 249-254.

George, S. \& Murali, V. (2005). Pathological gambling: an overview of assessment and treatment. Advances in Psychiatric Treatment, 11(6), 450-456.

Gunnell, D., Harbord, R., Singleton, N., Jenkins, R., \& Lewis, G. (2004). Factors influencing the development and amelioration of suicidal thoughts in the general population. The British Journal of Psychiatry, 185(5), 385393.

Harris, E. C. \& Barraclough, B. (1997). Suicide as an outcome for mental disorders. A meta-analysis. The British Journal of Psychiatry, 170(3), 205-228.

Hawton, K. \& van Heeringen, K. (2009). Suicide. The Lancet, 373, 13721381.

Hodgins, D. C., Mansley, C. \& Thygesen, K. (2006). Risk factors for suicide ideation and attempts among pathological gamblers. The American Journal on Addictions, 15(4), 303-310.

IBM Corp. Released 2013. IBM SPSS Statistics for Windows, Version 22.0. Armonk, NY: IBM Corp

Kessler, R. C., Berglund, P., Borges, G., Nock, M. \& Wang, P. S. (2005). Trends in suicide ideation, plans, gestures, and attempts in the United States, 1990-1992 to 2001-2003. The Journal of the American Medical Association, 293(20), 2487-2495.

Kessler, R. C., Borges, G. \& Walters, E. E. (1999). Prevalence of and risk factors for lifetime suicide attempts in the National Comorbidity Survey. Archives of General Psychiatry, 56(7), 617-626.

Kessler, R. C., Greenberg, P. E., Mickelson, K. D., Meneades, L. M., \& Wang, P. S. (2001). The effects of chronic medical conditions on work loss and work cutback. Journal of Occupational and Environmental Medicine, 43(3), 218-225. 
Kroenke, K., Spitzer, R. L. \& Williams, J. B. (2001). The PHQ- 9. Journal of General Internal Medicine, 16(9), 606-613.

Ledgerwood, D. M. \& Petry, N. M. (2004). Gambling and suicidality in treatment-seeking pathological gamblers. The Journal of Nervous and Mental Disease, 192(10), 711-714.

Kim, S. W., Grant, J. E., Eckert, E. D., Faris, P. L. \& Hartman, B. K. (2006). Pathological gambling and mood disorders: Clinical associations and treatment implication. Journal of Affective Disorders, 92,109-116.

Maccallum, F. \& Blaszczynski, A. (2003). Pathological gambling and suicidality: An analysis of severity and lethality. Suicide and LifeThreatening Behavior, 33(1), 88-98.

Manning, V., Koh, P. K., Yang, Y., Ng, A., Guo, S., Kandasami, G., et al. (2015). Suicidal ideation and lifetime attempts in substance and gambling disorders. Psychiatry Research, 255, 706-709.

McCormick, R. A., Russo, A. M., Ramirez, L. F. \& Taber, J. I. (1984). Affective disorders among pathological gamblers seeking treatment. The American Journal of Psychiatry, 141, 215-218.

Newman, S. C. \& Thompson A. H. (2003). A Population-Based Study of the Association Between Pathological Gambling and Attempted Suicide. Suicide and Life-Threatening Behavior, 33, 80-87.

Nock, M. K., Borges, G., Bromet, E. J., Alonso, J., Angermeyer, M., Beautrais, A., et al. (2008). Cross-national prevalence and risk factors for suicidal ideation, plans and attempts. The British Journal of Psychiatry, 192(2), 98-105.

Oquendo, M. A., Currier, D. \& Mann, J. J. (2006). Prospective studies of suicidal behavior in major depressive and bipolar disorders: What is the evidence for predictive risk factors? Acta Psychiatrica Scandinavica, 114(3), 151-158.

Petry, N. M. \& Kiluk, B. D. (2002). Suicidal ideation and suicide attempts in treatment-seeking pathological gamblers. The Journal of Nervous and Mental Disease, 190(7), 462.

Pridmore, S. \& Reddy, A. (2012). Financial loss and suicide. Malaysian Journal of Medical Sciences, 19, 74-76.

Qin, P., Agerbo, E. \& Mortensen, P. B. (2002). Suicide risk in relation to family history of completed suicide and psychiatric disorders: a nested case-control study based on longitudinal registers. The Lancet, 360, 1126-1130. 
Rudd, M. D. \& Joiner, T. (1998). The assessment management and treatment of suicidality: Toward clinically informed and balanced standards of care. Clinical Psychology: Science and Practice, 5(2), 135150.

Ruengorn, C., Sanichwankul, K., Niwatananun, W., Mahatnirunkul, S., Pumpaisalchai, W. \& Patumanond, J. (2011). Incidence and risk factors of suicide reattempts within 1 year after psychiatric hospital discharge in mood disorder patients. Clinical Epidemiology, 3, 305-313.

Scowcroft, E. (2016). Samaritans: suicide statistics report 2016.Retrieved from

http://www.samaritans.org/sites/default/files/kcfinder/files/Samaritans\%20 suicide\%20statistics\%20report\%202016.pdf

Séguin, M., Boyer, R., Lesage, A., McGirr, A., Suissa, A., Tousignant, M., et al. (2010). Suicide and gambling: Psychopathology and treatmentseeking. Psychology of Addictive Behaviors, 24, 541-547.

Specker, S. M., Carlson, G. A., Edmonson, K. M., Johnson, P. E. \& Marcotte, M. (1996). Psychopathology in pathological gamblers seeking treatment. Journal of Gambling Studies, 12(1), 67-81.

Spitzer, R. L., Kroenke, K., Williams, J. B., \& Löwe, B. (2006). A brief measure for assessing generalized anxiety disorder: the GAD-7. Archives of Internal Medicine, 166(10), 1092-1097.

Stanley, B. \& Brown, G. K. (2012). Safety planning intervention: A brief intervention to mitigate suicide risk. Cognitive and Behavioral Practice, 19(2), 256-264.

Thon, N., Preuss, U. W., Pölzleitner, A., Quantschnig, B., Scholz, H., Kühberger, A., et al. (2014). Prevalence of suicide attempts in pathological gamblers in a nationwide Austrian treatment sample. General Hospital Psychiatry, 36(3), 342-346.

Toneatto, T., \& Pillai, S. (2016). Mood and Anxiety Disorders Are the Most Prevalent Psychiatric Disorders among Pathological and Recovered Gamblers. International Journal of Mental Health and Addiction, 14(3), 217-227.

Wardle, H., Moody, A., Spence, S., Orford, J., Volberg, R., Jotangia, D., et al. (2010). British Gambling Prevalence Survey. London: National Centre for Social Research.

Windfuhr, K. \& Kapur, N. (2011). Suicide and mental illness: A clinical review of 15 years findings from the UK National Confidential Inquiry into Suicide. British Medical Bulletin, 100, 101-121. 
Weissman, M. M., Bland, R. C., Canino, G. J., Greenwald, S., Hwu, H. G., Joyce, P. R., et al. (1999). Prevalence of suicide ideation and suicide attempts in nine countries. Psychological Medicine, 29(1), 9-17. 